\title{
EVALUACIÓN PRELIMINAR DE LA CALIDAD DE LA ESCORRENTÍA PLUVIAL SOBRE TEJADOS PARA SU POSIBLE APROVECHAMIENTO EN ZONAS PERIURBANAS DE BOGOTÁ
}

\section{PRELIMINARY ASSESSMENT OF ROOF RUNOFF RAIN WATER QUALITY FOR POTENTIAL HARVESTING IN BOGOTA'S PERI-URBAN AREAS}

\begin{abstract}
Andrés Torres ${ }^{1}$, Sandra Méndez-Fajardo², Liliana López-Kleine³ ${ }^{3}$ Valentina Marín ${ }^{4}$, Jorge Andrés González ${ }^{5}$ Juan Camilo Suárez ${ }^{6}$, Julián David Pinzón ${ }^{7}$, Alejandra Ruiz ${ }^{8}$

${ }^{1}$ Ingeniero Civil, Especialista en Sistemas Gerenciales de Ingeniería, M.Sc. en Ingeniería Civil y Ph.D. en Ingeniería Civil con énfasis en Hidrología Urbana. Grupo de Investigación ISAD, Facultad de Ingeniería, Pontificia Universidad Javeriana, Calle 40 No. 5-50, Edif. José Gabriel Maldonado, S.J., Bogotá D.C., Colombia, andres.torres@javeriana.edu.co ${ }^{2}$ Ingeniera Civil y M.Sc en Ingeniería Civil con énfasis en Ingeniería Ambiental. Grupo de Investigación ISAD, Facultad de Ingeniería, Pontificia Universidad Javeriana, Calle 40 No. 5-50, Edif. José Gabriel Maldonado, S.J., Bogotá D.C., Colombia, sandra.mendez@javeriana.edu.co ${ }^{3}$ Bióloga, M.Sc. en Ecología, Evolución y Biometría y Ph.D. en Ciencias Biológicas. Departamento de Estadística, Facultad de Ciencias, Universidad Nacional de Colombia, sede Bogotá, Colombia, llopezk@unal.edu.co 4, 5, 6,7 Estudiante de Ingeniería Civil. Facultad de Ingeniería, Pontificia Universidad Javeriana, Calle 40 No. 5-50, Edif. José Gabriel Maldonado, S.J., Bogotá D.C., Colombia, marinv@javeriana. edu.co, jgonzalezv@javeriana.edu.co, jsuarezb@javeriana.edu.co, julian.pinzon@javeriana.edu.co ${ }^{8}$ Ingeniera Civil y estudiante de Maestría en Hidrosistemas. Facultad de Ingeniería, Pontificia Universidad Javeriana, Calle 40 No. 5-50, Edif. José Gabriel Maldonado, S.J., Bogotá D.C., Colombia, alejandra.ruiz.l@javeriana.edu.co
\end{abstract}

Rev. U.D.C.A Act. \& Div. Cient. 14(1): 127 - 135, 2011

\section{RESUMEN}

En Colombia, varias comunidades, cuyo acceso al servicio de agua potable es limitado o precario, recolectan aguas lluvias para diferentes usos domésticos. Este artículo presenta los resultados de análisis de calidad de aguas lluvias de escorrentía sobre tejados, en barrios de Kennedy (Bogotá) y del municipio de Soacha (Cundinamarca), con miras a evaluar su adaptabilidad para satisfacer usos domésticos, en dichas comunidades. De acuerdo a los resultados obtenidos, el agua muestreada no es apta para ninguno de los usos de las comunidades estudiadas, debido principalmente a altos valores de turbiedad y altas concentraciones de Sólidos Suspendidos Totales, Demanda Bioquímica de Oxígeno a los cinco días y metales pesados; sin embargo, se detectó una alta variabilidad espacial y temporal de los resultados, así como en función de los materiales de los techos de las casas. En algunos casos y condiciones especiales, el agua lluvia de escorrentía sobre tejados, se podría adaptar para ser utilizada como fuente alternativa para satisfacer algunos usos domésticos.

Palabras clave: Aprovechamiento de aguas lluvias, calidad de aguas lluvias urbanas, corrosión de tejados.

\section{SUMMARY}

In Colombia, several communities with limited or precarious access to potable water services collect rain water for different domestic uses. This paper presents results of the quality analysis of runoff rainwater on roofs in Kennedy (Bogotá) and Soacha (Cundinamarca) in order to evaluate their adaptability to satisfy domestic uses in these districts. Based on the results obtained, it can be conclude that the sampled water is not suitable for none of the possible domestic uses in these communities. This is due to high values of turbidity and high concentrations of Total Suspended Solids, Biochemical Oxygen Demand and heavy metals. Nevertheless, high spatial 
and temporal variability was detected, as well as variability in function of the roof material. In some of the analyzed samples, the runoff water of the roofs could be adapted as an alternative for domestic uses.

Key words: Urban stormwater quality, stormwater harvesting, roof corrosion.

\section{INTRODUCCIÓN}

Hoy en día existe una atención creciente en el aprovechamiento del agua lluvia como fuente potencial de agua en las ciudades (Hatt et al. 2006; Fletcher et al. 2007). En especial, en países en desarrollo, el aprovechamiento de aguas lluvias se ha convertido, en los últimos años, en una alternativa interesante, debida, principalmente, al bajo costo de operación asociado. Así, por ejemplo, se han reportado experiencias exitosas de aprovechamiento de aguas lluvias en países en desarrollo, tanto de Asia (Han $\mathcal{E} \mathrm{Ki}, 2010$ ) como de Latinoamérica (Ghisi et al. 2009); sin embargo, internacionalmente, se reconoce que la implementación de estos sistemas depende no solamente de la cantidad de lluvia u oferta hídrica disponible en un determinado lugar, sino también de la calidad de ésta y de los usos que se le quieran dar (Mitchell et al. 2008). En efecto, las aguas lluvias urbanas contienen numerosos contaminantes que pueden afectar la salud humana y la calidad de las aguas superficiales y subterráneas (Göbel et al. 2007; McCarthy et al. 2008). En la literatura, se reporta que las concentraciones típicas en áreas urbanas de metales presentes en las aguas de escorrentía, como resultado de la corrosión de las superficies de los tejados, varían de $0,1 \mu \mathrm{g} / \mathrm{L}$ a $32 \mu \mathrm{g} / \mathrm{L}$, para $\mathrm{Cd}$; de $3 \mu \mathrm{g} / \mathrm{L}$ a $247 \mu \mathrm{g} / \mathrm{L}$, para Cu; de $16 \mu \mathrm{g} / \mathrm{L}$ a $2764 \mu \mathrm{g} / \mathrm{L}$, para $\mathrm{Pb}$ y de $802 \mu \mathrm{g} / \mathrm{L}$ a $38061 \mu \mathrm{g} / \mathrm{L}$, para Zn (Gromaire-Mertz et al. 1999; Göbel et al. 2007). Estos metales provienen de partículas de polvo como resultado de procesos de combustión, industrias de metales ferrosos y no ferrosos, plantas de incineración, industrias cementeras o vidrieras y tráfico vehicular (Göbel et al. 2007). Se ha hallado, además, que en aguas de escorrentía sobre superficies de tejados se encuentran concentraciones de Sólidos Suspendidos Totales (SST), entre 3mg/L y 304mg/L, Demanda Bioquímica de Oxígeno a los cinco días $\left(\mathrm{DBO}_{5}\right)$ entre $1 \mathrm{mg} / \mathrm{L}$ y $27 \mathrm{mg} / \mathrm{L}$ (Gromaire-Mertz et al. 1999) y valores bajos de $\mathrm{pH}$ (entre 4,7 y 6,8 según Göbel et al. 2007).

Varios factores influyen en la corrosión de los tejados, entre los que se encuentran la humedad relativa y el $\mathrm{pH}$ de la lluvia (Gromaire-Mertz et al. 1999). Así mismo, se ha encontrado que la concentración de contaminantes presentes en las aguas de escorrentía urbana depende también de depósitos atmosféricos en tiempo seco y en tiempos de lluvia, de las características de la superficie y del tipo de zona urbana (Gromaire-Mertz et al. 1999; Schueler \& Holland, 2000; Göbel et al. 2007). Adicionalmente, el proceso de escorrentía está estrechamente relacionado con las características de las precipitaciones, como el volumen, la intensidad, el caudal y los periodos de tiempo seco anterior.

En Colombia, varias comunidades recolectan aguas lluvias para diferentes usos domésticos, como desagüe de inodoros, lavado de patios, pisos y fachadas, riego y consumo animal y humano. Esta práctica es común en aquellas comunidades con un acceso limitado o precario al servicio de agua potable o cuando el costo del servicio no es adaptado al nivel de ingresos de los habitantes. Específicamente, de acuerdo con observaciones directas y salidas de campo a zonas periurbanas de Bogotá, realizadas desde el 2002, varias familias están, actualmente, empleando el agua lluvia de escorrentía sobre tejados, para satisfacer algunas prácticas domésticas sin evaluar, previamente, su calidad y, por lo tanto, existe un riesgo sanitario asociado a dicha explotación y, en particular, debido a la calidad del agua lluvia y su adaptabilidad a las diferentes actividades de las comunidades y a los métodos de recolección, de almacenamiento, de conservación y de distribución. A pesar de dichos riesgos, en Colombia no se han reportado aún resultados de estudios detallados sobre la calidad de aguas lluvias de escorrentía sobre tejados ni su variabilidad, con el fin de evaluar su adaptabilidad a diferentes usos por parte de las comunidades. El objetivo de este trabajo consistió en caracterizar el agua lluvia de escorrentía sobre tejados para su eventual uso doméstico, en zonas periurbanas de Bogotá, a través de los casos específicos de estudio de los barrios Altos de la Florida (Soacha, Cundinamarca), Villa Alexandra y Acacias (localidad de Kennedy, Bogotá), donde es práctica común la utilización de las aguas lluvias de escorrentía sobre los tejados.

\section{MATERIALES Y MÉTODOS}

Soacha, se ha constituido como un territorio de concentración de actividades industriales, como textil, fabricación de productos químicos, pinturas y barnices, jabones y detergentes, caucho, vidrio, ladrillos, fundición de cobre, plomo y acero (Pachón Quinche, 2005). Por otro lado, la localidad de Kennedy y, en particular, el barrio Villa Alexandra, cuenta con bodegas que son utilizadas como talleres. Dentro de la zona concurre, también, el negocio de materiales reciclables, así como quema de combustibles domésticos y basuras (llantas, plásticos y residuos sólidos en general) y fabricación de carbón vegetal. Aunque no se tienen mediciones específicas que permitan estimar la magnitud, acaece una contaminación atmosférica en la 
zona, provocada, principalmente, por el tráfico vehicular (emanación de gases y levantamiento de polvo de las calles no pavimentadas). En estos dos sitios es práctica común, por parte de las comunidades que los habitan, la utilización de las aguas lluvias recolectadas después de su escorrentía sobre los tejados de las viviendas.

Entre febrero y abril de 2010, se recolectaron y se analizaron siete muestras en el barrio Altos de la Florida (Soacha) y 23 muestras en Villa Alexandra y Acacias (Localidad de Kennedy), provenientes de casas con tejas de zinc y fibrocemento. La diferencia en el número de muestras, se debe a la escasa precipitación existente en Soacha, en comparación con aquella observada en la localidad de Kennedy. Sobre dichas muestras, se determinaron valores de $\mathrm{pH}$, color verdadero y turbiedad y concentraciones de SST, $\mathrm{DBO}_{5}$ y metales pesados (Cd, Cu, Pb y Zn), en el Laboratorio de Pruebas y Ensayos, de la Facultad de Ingeniería, de la Pontificia Universidad Javeriana, sede Bogotá, siguiendo los procedimientos indicados en los métodos estándar (APHA/AWWA/WEF, 1998). Se decidió analizar cada parámetro de calidad por triplicado, de tal manera que se calculara la incertidumbre experimental proveniente, tanto de la precisión de los aparatos utilizados como del submuestreo y manipulación de muestras y equipos de laboratorio, así como facilitar el análisis de varianza previsto sobre los resultados obtenidos. Con el fin de evaluar la eventual manejo de las aguas de escorrentía muestreadas en diferentes usos domésticos, como desagüe de inodoros, lavado de patios, pisos y fachadas, riego y consumo animal y humano, se revisaron estándares de calidad y documentos relacionados, a nivel nacional (Ministerio de Salud República de Colombia, 1984; Ministerio de la Protección Social, Ministerio de Ambiente Vivienda y Desarrollo Territorial, 2007) e internacional (European Union, 1998; U.S. EPA, 2004; WHO, 2006; European Union, 2006). Dichos estándares y documentos proponen una calidad mínima del agua recolectada para diferentes usos, teniendo en cuenta parámetros microbiológicos y fisicoquímicos.

Tras realizar pruebas de homogeneidad de varianza (test de Bartlett) y de normalidad (test de Shapiro-Wilk) a los resultados de calidad de aguas obtenidos, se concluyó que en todos los casos se debía realizar pruebas de Kruskal-Wallis, ya que en ningún caso se obtuvo homogeneidad de varianza ni normalidad (valores $p<0,05$ ). Las pruebas de KruskalWallis para cada parámetro de calidad medido, se realizaron con el propósito de identificar la influencia del tipo de tejado (tejas de zinc o fibrocemento), de la variabilidad de los eventos lluviosos y del tipo de zona urbana, sobre la calidad del agua muestreada. Con el fin de observar eventuales correlaciones entre las diferentes variables medidas, se llevaron a cabo pruebas de correlación de Pearson (para muestras con distribución normal) y de Spearman. Este análisis estadístico, se realizó utilizando el programa $\mathrm{R}(\mathrm{R}$ Development Core Team, 2010).

\section{RESULTADOS Y DISCUSIÓN}

A manera de síntesis, las tablas 1 a 3 muestran los valores promedio de contaminantes y de desviaciones estándar para cada localidad (Soacha y Kennedy), cada evento lluvioso (15 de febrero, 16 de febrero, 12 de marzo, 22 de marzo, 12 de abril y 30 de abril de 2010) y cada material de tejado (tejas de fibrocemento, tejas de fibrocemento pintadas y tejas de zinc y tejas de zinc), respectivamente. De acuerdo a los resultados obtenidos, el agua muestreada no es apta para vaciar sanitarios y orinales, riego, lavado de pisos y fachadas ni consumo humano. Lo anterior, se debe, principalmente, a: (i) altos valores de turbiedad para los tres primeros eventos lluviosos (en particular para las muestras del 15 de febrero, 16 de febrero y 12 de marzo: Tabla 2), superiores a 5 NTU, el cual, es un valor límite recomendado para los usos menos restrictivos, como riego no agrícola (U.S. EPA, 2004); (ii) altas concentraciones en SST para los tres primeros eventos lluviosos (en particular para las muestras del 15 de febrero, 16 de febrero y 12 de marzo: Tabla 2), superiores a 30mg/L, un valor límite recomendado para los usos menos restrictivos, como riego agrícola superficial (U.S. EPA, 2004); (iii) altas concentraciones en $\mathrm{DBO}_{5}$, en especial, para los eventos del 15 de febrero, 16 de febrero y 22 de marzo (Tabla 2), con valores superiores a $10 \mathrm{mg} / \mathrm{L}$, el cual, es el valor límite recomendado para descarga de sanitarios y de orinales, usos recreativos con contacto primario y riego (U.S. EPA, 2004); (iv) altas concentraciones en Cd para todas las muestras analizadas (Tabla 2), superiores a 0,003 mg/L, valor límite recomendado para consumo humano (Ministerio de la Protección Social, Ministerio de Ambiente Vivienda y Desarrollo Territorial, 2007); (v) altas concentraciones en Cd para los tres últimos eventos lluviosos (22 de marzo, 12 de abril y 30 de abril: tabla 2), superiores a 0,01mg/L, el cual, es un valor límite recomendado para riego agrícola (Ministerio de Salud República de Colombia, 1984; U.S. EPA, 2004) y, en particular, si se quiere evitar el taponamiento de elementos de irrigación; (vi) altas concentraciones en $\mathrm{Pb}$, superiores a 0,01 mg/L, valor límite recomendado para consumo humano (Ministerio de Salud República de Colombia, 1984; Ministerio de la Protección Social, Ministerio de Ambiente Vivienda y Desarrollo Territorial, 2007); (vii) altas concentraciones en Zn, en especial, en casas con techos de zinc, superiores a $2 \mathrm{mg} / \mathrm{L}$ (Tabla 3), el cual, es un valor límite recomendado para riego (Ministerio de Salud República de Colombia, 1984; U.S. EPA, 2004). Adicional a lo anterior, se detectaron valores un poco elevados de $\mathrm{pH}$, superiores a siete unidades, lo que restringe su uso para riego, debido a un posible taponamiento de los elementos de irrigación (Lazarova et al. 2004). 
Con excepción de los metales, se detectó una mejor calidad del agua de las muestras provenientes de los dos últimos eventos lluviosos muestreados (12 y 30 de abril), tanto en Kennedy como en Soacha. Estas observaciones, se podrían deber a un cambio en el régimen (época seca/época lluviosa) y/o características de las lluvias (altura, intensidad, duración de lluvias, duración de tiempo seco anterior) de abril, con respecto de febrero a marzo o a una disminución de la contaminación en abril. En caso de que se llegara a comprobar que la mejoría en la calidad de las aguas lluvias de escorrentía sobre tejados, como aquella observada durante abril con respecto a los meses anteriores, está asociada a una época de lluvias, se podría plantear una utilización de las aguas lluvias recolectadas durante estas épocas para vaciar sanitarios y orinales o para lavado de pisos y fachadas.

De las pruebas de Kruskal-Wallis realizadas sobre los resultados de color, se pudo concluir que, tanto la variabilidad temporal (factor etiquetado como "evento") como de material de los techos considerados (factor etiquetado como "techo"), tienen una influencia significativa sobre los resultados obtenidos (valores $p<0,05$ ). Cabe anotar que este parámetro sólo fue medido en Soacha y, por lo tanto, no se contempla una variabilidad de los resultados en función de la localización espacial. Un análisis más detallado de los

Tabla 1. Valores promedio de contaminantes y desviaciones estándar (entre paréntesis), obtenidos durante los eventos lluviosos del 15 de febrero al 30 de abril de 2010. (NA: el parámetro no se midió; *: el valor reportado corresponde a la mediana).

\begin{tabular}{|l|c|c|}
\hline \multicolumn{1}{|c|}{ CONTAMINANTE } & SOACHA & KENNEDY \\
\hline color (UPC) & $\begin{array}{c}39 \\
(36)\end{array}$ & NA \\
\hline $\mathrm{pH}^{*}$ & 7,4 & 8,0 \\
\hline turbiedad (NTU) & 17 & 21 \\
& $(20)$ & $(19)$ \\
\hline $\mathrm{SST}(\mathrm{mg} / \mathrm{L})$ & 149 & 124 \\
& $(138)$ & $(168)$ \\
\hline DBO 5 (mg/L) & $\mathrm{NA}$ & $\begin{array}{c}20 \\
(16)\end{array}$ \\
\hline Cd (mg/L) & 0,3835 & 0,1725 \\
& $(0,2013)$ & $(0,3137)$ \\
\hline $\mathrm{Cu}(\mathrm{mg} / \mathrm{L})$ & 0,0589 & 0,0349 \\
& $(0,0132)$ & $(0,0243)$ \\
\hline $\mathrm{Pb}(\mathrm{mg} / \mathrm{L})$ & 0,3106 & 0,2273 \\
& $(0,1063)$ & $(0,0545)$ \\
\hline $\mathrm{Zn}(\mathrm{mg} / \mathrm{L})$ & 2,0880 & 2,4650 \\
& $(1,9049)$ & $(1,6485)$ \\
\hline
\end{tabular}

resultados de las pruebas de Kruskal-Wallis indica que el factor que más influencia tiene sobre el color es la variabilidad de los eventos lluviosos, ya que el porcentaje de varianza correspondiente sobre la varianza total es mayor para este factor que para los otros factores considerados (Figura 1); sin embargo, este resultado se concluye a partir de tan sólo ocho muestras tomadas en Soacha, durante dos eventos lluviosos y, por lo tanto, es bastante prematuro formular conclusiones definitivas. Parece necesario, entonces, tener un mayor número de muestras para diferentes eventos lluviosos y localidades. En la tabla 2 , se observa que la muestra correspondiente al evento del 12 de marzo presenta valores de color mayores que aquellos del evento del $30 \mathrm{de}$ abril. A partir de una comparación de los resultados mediante una prueba $t$ con ajuste de Bonferroni, se logró concluir que dicha diferencia es significativa (valor $p<0,05$ ). Estas diferencias, se podrían deber a un cambio en el régimen de lluvias de abril con respecto a marzo y, en particular, un periodo de tiempo seco anterior más prolongado, para el evento del 12 de marzo.

De las pruebas de Kruskal-Wallis realizadas sobre los resultados de $\mathrm{pH}$, se concluyó que tanto la variabilidad espacial (factor etiquetado como "localidad") como temporal (factor etiquetado como "evento") y de material de los techos considerados (factor etiquetado como "techo") tienen una influencia significativa sobre los resultados obtenidos (valores $p<0,05)$. Un análisis más detallado de los resultados de las pruebas de Kruskal-Wallis indica que el factor que más influencia tiene sobre el resultado de $\mathrm{pH}$ es el material del techo, ya que el porcentaje de varianza correspondiente sobre la varianza total es mayor para este factor que para los otros factores considerados (Figura 1). En la tabla 3, se observa que las muestras asociadas a los techos de zinc presentan valores de $\mathrm{pH}$ más bajos que los techos con tejas de fibrocemento. A partir de una comparación de los resultados mediante pruebas $t$ con ajuste de Bonferroni, se consiguió concluir que dichas diferencias son significativas (valores $p<0,05$ ).

De las pruebas de Kruskal-Wallis efectuadas sobre los resultados de turbiedad, SST y $\mathrm{DBO}_{5}$, se pudo concluir que la variabilidad temporal (factor etiquetado como "evento") es el único factor que tiene una influencia significativa sobre los resultados obtenidos (valores $p<0,05$ ) (Figura 1). En la tabla 2 , se detalla que las muestras recolectadas durante los eventos del 15 y 16 de febrero y 12 de marzo presentan valores de turbiedad y SST mayores, que aquellos obtenidos en los eventos posteriores. Además, se observa que la muestra recolectada durante el evento del 15 de febrero presenta valores de $\mathrm{DBO}_{5}$ mayores, que aquellos de los eventos posteriores. A partir de una comparación de los resultados mediante pruebas $t$ con ajuste de Bonferroni, se logró 
Tabla 2. Valores promedio de contaminantes y desviaciones estándar (entre paréntesis), obtenidos durante los eventos lluviosos del 15 de febrero al 30 de abril de 2010. (NA: el parámetro no se midió o no es posible calcular la desviación estándar; *: el valor reportado corresponde a la mediana).

\begin{tabular}{|c|c|c|c|c|c|c|}
\hline \multirow{2}{*}{ CONTAMINANTE } & \multicolumn{6}{|c|}{ FECHA DEL EVENTO (dd/mm) } \\
\hline & $15 / 02$ & $16 / 02$ & $12 / 03$ & 22/03 & $12 / 04$ & $30 / 04$ \\
\hline $\begin{array}{c}\text { color verdadero } \\
\text { (UPC) }\end{array}$ & NA & NA & $\begin{array}{c}78 \\
(21)\end{array}$ & NA & NA & $\begin{array}{c}15 \\
(15)\end{array}$ \\
\hline $\mathrm{pH}^{*}$ & 8,1 & 8,2 & 8,0 & 8,0 & 7,8 & 7,1 \\
\hline turbiedad (NTU) & $\begin{array}{c}45 \\
(11) \\
\end{array}$ & $\begin{array}{c}27 \\
(12)\end{array}$ & $\begin{array}{l}38 \\
(9) \\
\end{array}$ & $\begin{array}{c}5 \\
(9) \\
\end{array}$ & $\begin{array}{l}2 \\
(3) \\
\end{array}$ & $\begin{array}{c}4 \\
(6) \\
\end{array}$ \\
\hline SST (mg/L) & $\begin{array}{c}275 \\
(109)\end{array}$ & $\begin{array}{l}107 \\
(75) \\
\end{array}$ & $\begin{array}{c}269 \\
(125)\end{array}$ & $\begin{array}{c}78 \\
(155) \\
\end{array}$ & $\begin{array}{l}10 \\
(3)\end{array}$ & $\begin{array}{c}78 \\
(156) \\
\end{array}$ \\
\hline $\mathrm{DBO}_{5}(\mathrm{mg} / \mathrm{L})$ & $\begin{array}{c}47 \\
(24)\end{array}$ & $\begin{array}{l}19 \\
(7) \\
\end{array}$ & NA & $\begin{array}{l}19 \\
(5) \\
\end{array}$ & $\begin{array}{c}9 \\
(1) \\
\end{array}$ & NA \\
\hline $\mathrm{Cd}(\mathrm{mg} / \mathrm{L})$ & $\begin{array}{c}0,0236 \\
(0,0227)\end{array}$ & $\begin{array}{c}0,0137 \\
(0,0146)\end{array}$ & $\begin{array}{l}0,0206 \\
\text { (NA) }\end{array}$ & $\begin{array}{c}0,3604 \\
(0,2031)\end{array}$ & $\begin{array}{c}0,3539 \\
(0,1189)\end{array}$ & $\begin{array}{c}0,4742 \\
(0,2763)\end{array}$ \\
\hline $\mathrm{Cu}(\mathrm{mg} / \mathrm{L})$ & $\begin{array}{c}0,0329 \\
(0,0117)\end{array}$ & $\begin{array}{c}0,0340 \\
(0,0160)\end{array}$ & $\begin{array}{c}0,0400 \\
(0,0271)\end{array}$ & $\begin{array}{c}0,0347 \\
(0,0120)\end{array}$ & $\begin{array}{c}0,0378 \\
(0,0156)\end{array}$ & $\begin{array}{c}0,0703 \\
(0,0155) \\
\end{array}$ \\
\hline $\mathrm{Pb}(\mathrm{mg} / \mathrm{L})$ & $\begin{array}{c}0,2200 \\
(0,0855)\end{array}$ & $\begin{array}{c}0,2345 \\
(0,1320)\end{array}$ & $\begin{array}{c}0,3106 \\
(0,0545)\end{array}$ & NA & NA & NA \\
\hline Zn (mg/L) & $\begin{array}{c}3,7530 \\
(1,5711)\end{array}$ & $\begin{array}{c}2,7680 \\
(2,1856)\end{array}$ & $\begin{array}{c}2,5650 \\
(2,4211)\end{array}$ & $\begin{array}{c}2,0210 \\
(2,0642)\end{array}$ & $\begin{array}{c}1,2460 \\
(1,1104)\end{array}$ & $\begin{array}{c}1,8020 \\
(1,2456)\end{array}$ \\
\hline
\end{tabular}

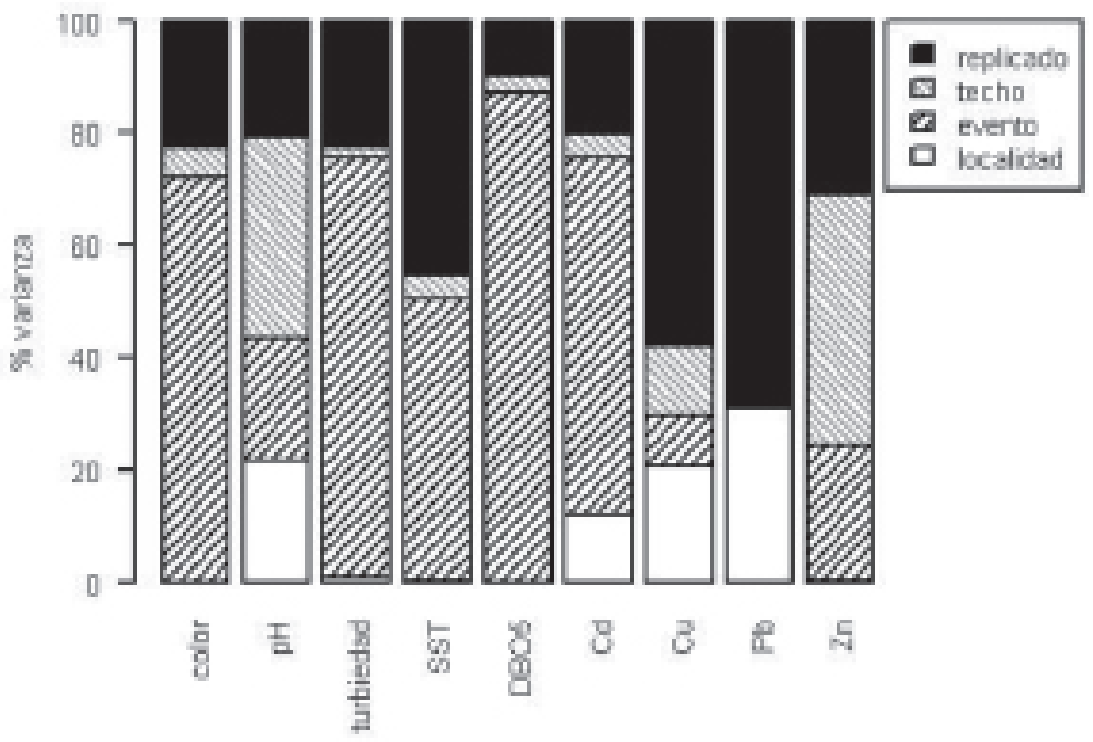

Figura 1. Resultados de análisis de varianza para cada parámetro de calidad medido. 
Tabla 3. Valores promedio de contaminantes y desviaciones estándar (entre paréntesis), obtenidos para cada material de tejado (FC: fibrocemento; FCp-Zn: fibrocemento pintadas y tejas de zinc; Zn: tejas de zinc), durante los eventos lluviosos del 15 de febrero al 30 de abril de 2010. (NA: el parámetro no se midió o no es posible calcular la desviación estándar; *: el valor reportado corresponde a la mediana).

\begin{tabular}{|l|c|c|c|}
\hline CONTAMINANTE & FC & FCp-Zn & Zn \\
\hline \multirow{2}{*}{ color (UPC) } & $\begin{array}{c}95 \\
(\mathrm{NA})\end{array}$ & $\begin{array}{c}25 \\
(22)\end{array}$ & $\begin{array}{c}45 \\
(57)\end{array}$ \\
\hline $\mathrm{pH}^{*}$ & 8,3 & 7,2 & 7,8 \\
\hline \multirow{2}{*}{ turbiedad (NTU) } & 24 & 11 & 19 \\
& $(22)$ & $(14)$ & $(18)$ \\
\hline \multirow{2}{*}{$\mathrm{SST}(\mathrm{mg} / \mathrm{L})$} & 104 & 141 & 156 \\
& $(107)$ & $(182)$ & $(169)$ \\
\hline \multirow{2}{*}{$\mathrm{DBO}_{5}(\mathrm{mg} / \mathrm{L})$} & 21 & $\mathrm{NA}$ & 19 \\
& $(21)$ & 0,4516 & 0,1487 \\
$\mathrm{C} \mathrm{Cd}(\mathrm{mg} / \mathrm{L})$ & 0,1892 & $0,3167)$ & $(0,1720)$ \\
\hline \multirow{2}{*}{$\mathrm{Cu}(\mathrm{mg} / \mathrm{L})$} & $(0,2234)$ & $(0,310)$ \\
\hline \multirow{2}{*}{$\mathrm{Pb}(\mathrm{mg} / \mathrm{L})$} & 0,0409 & 0,0594 & 0,0342 \\
& $(0,0122)$ & $(0,0320)$ & $(0,0164)$ \\
\hline \multirow{2}{*}{$\mathrm{Zn}(\mathrm{mg} / \mathrm{L})$} & 0,2458 & 0,2658 & 0,2390 \\
& $(0,0924)$ & $(\mathrm{NA})$ & $(0,1259)$ \\
\hline
\end{tabular}

concluir que dichas diferencias son significativas (valores $p$ $<0,05$ ), tanto para los valores de turbiedad como de SST y $\mathrm{DBO}_{5}$. Estas diferencias, se podrían deber a un cambio en el régimen de lluvias de abril con respecto a febrero y marzo y, en particular, en lo que respecta los periodos de tiempo seco anteriores a las lluvias: un periodo prolongado de tiempo seco anterior a una lluvia podría favorecer la acumulación de material particulado sobre los techos, el cual, podría ser arrastrado, posteriormente, durante el evento lluvioso. Para el caso de la $\mathrm{DBO}_{5}$, estas diferencias se podrían deber a una acumulación de materia orgánica, como por ejemplo, hojas y excremento de aves antes del evento del 15 de febrero, posiblemente, durante un periodo prolongado de tiempo seco anterior a dicho evento. Cabe anotar que la DBO5 sólo fue medida en la localidad de Kennedy y, por lo tanto, no se contempla una variabilidad de los resultados en función de la localización espacial.

De las pruebas de Kruskal-Wallis generadas sobre los resultados de $\mathrm{Cd}$ y $\mathrm{Cu}$, se concluyó que tanto la variabilidad espacial (factor etiquetado como "localidad"), como temporal (factor etiquetado como "evento") y de material de los techos considerados (factor etiquetado como "techo") tienen una influencia significativa sobre los resultados obtenidos (valores $p<0,05$ ). Un análisis más detallado de los resultados de las pruebas de Kruskal-Wallis indica que el factor que más influencia tiene sobre el resultado de $\mathrm{Cd}$ es la variabilidad de los eventos lluviosos, ya que el porcentaje de varianza correspondiente sobre la varianza total es mayor para este factor que para los otras variables consideradas (Figura 1). En la tabla 2, se indica que las muestras recolectadas durante los eventos del 15 y 16 de febrero y 12 de marzo presentan valores de Cd inferiores a aquellos obtenidos para las pruebas recolectadas durante los eventos posteriores. Además, se observa que las muestras recogidas durante el evento del 30 de abril presentan valores de $\mathrm{Cu}$ superiores a aquellos obtenidos para los eventos anteriores. A partir de una comparación de los resultados mediante pruebas $t$ con ajuste de Bonferroni, se logró concluir que dichas diferencias son significativas (valores $p<0,05$ ). Estas diferencias representan un aumento de la disponibilidad de $\mathrm{Cd}$ y $\mathrm{Cu}$ en el agua de escorrentía sobre los tejados con respecto al tiempo. Esta disponibilidad podría estar ligada a un aumento en la intensidad o altura de las lluvias de abril con respecto a aquellas de febrero y marzo.

De las pruebas de Kruskal-Wallis desarrolladas sobre los resultados de $\mathrm{Pb}$, se consiguió deducir que, tanto la variabilidad espacial (factor etiquetado como "localidad") como temporal (factor etiquetado como "evento") tienen una influencia significativa sobre los resultados de $\mathrm{Pb}$ obtenidos (valores $p<0,05$ ).

Para SST, $\mathrm{Cu}$ y $\mathrm{Pb}$, la variabilidad de los replicados tiene una influencia muy importante sobre los resultados, ya que el porcentaje de varianza correspondiente sobre la varianza total es mayor que para los factores considerados (Figura 1). La observación anterior indicaría una presencia de problemas en los procesos experimentales de muestreo y/o análisis de laboratorio y, consecuentemente, se podría cuestionar la fiabilidad de los resultados obtenidos, lo cual, se deberá corregir en futuros estudios. Adicionalmente, cabe anotar que las concentraciones de $\mathrm{Pb}$, se determinaron únicamente para las muestras recolectadas durante los eventos lluviosos del 15 de febrero al 12 de marzo, ya que, después, se presentaron problemas técnicos con el equipo empleado, que no se lograron solucionar para el análisis de las muestras posteriores.

Como se mencionó anteriormente, para $\mathrm{Cd}, \mathrm{Cu}$ y $\mathrm{Pb}$, la variabilidad espacial (factor etiquetado como "localidad") tiene una influencia significativa sobre los resultados obtenidos (valores $p<0,05$ ). En efecto, se hallaron valores significativamente más altos de estos tres metales en Soacha que en Kennedy (Tabla 1). Esto, se puede deber, a que la actividad industrial en Soacha es bastante más desarrollada que en Kennedy, como se mencionó previamente. 
De las pruebas de Kruskal-Wallis realizadas sobre los resultados de $\mathrm{Zn}$, se pudo concluir que, tanto la variabilidad temporal (factor etiquetado como "evento") como de material de los techos considerados (factor etiquetado como "techo") poseen una influencia significativa sobre los resultados de Zn obtenidos (valores $p<0,05$ ). Un análisis más detallado de los resultados de las pruebas de Kruskal-Wallis indica que el factor que más influencia tiene sobre el resultado de $\mathrm{Zn}$ es el material de los techos, puesto que el porcentaje de varianza correspondiente sobre la varianza total es mayor para este factor que para los otros factores considerados (Figura 1). En la tabla 3, se detalla que las muestras asociadas con techos de fibrocemento presentan concentraciones de $\mathrm{Zn}$ inferiores a aquellas asociadas con techos de zinc. A partir de una comparación de los resultados usando pruebas $t$ con ajuste de Bonferroni, se consiguió determinar que dichas diferencias son significativas (valores $p<0,05$ ).

Para los resultados presentados en las tablas 1 a 3 , se efectuaron pruebas de correlación entre todos los parámetros. Dichas pruebas de correlación, se realizaron aplicando el método de Pearson (para muestras con distribución normal) y el método de Spearman. Los coeficientes de correlación, se presentan en la tabla 4 (arriba): coeficientes de correlación de Pearson encima de la diagonal y coeficientes de correlación de Spearman debajo de la diagonal. Los valores $p$ inferiores a 0,05 (lo que indica correlaciones significativas con un 95 $\%$ de confianza), se indican en la tabla 4 (abajo): valores $p$ de Pearson, encima de la diagonal y valores $p$ de Spearman, debajo de la diagonal.

Con respecto a las concentraciones en metales, los resultados expuestos en la tabla 4 sugieren que, aunque no de manera significativa, existen correlaciones negativas entre el $\mathrm{pH}$ y las concentraciones en metales ( $\mathrm{Cd}, \mathrm{Cu}, \mathrm{Pb}, \mathrm{Zn}$ ), lo que está de acuerdo con las observaciones reportadas en la literatura (Gromaire-Mertz et al. 1999). Adicionalmente, se observa que: (i) existen correlaciones significativas entre las concentraciones de $\mathrm{Zn}$ y los valores de turbiedad, SST y Cd; (ii) existe una correlación significativa entre las concentraciones de $\mathrm{Cd}$ y los valores de turbiedad; (iii) existe una correlación significativa entre las concentraciones de $\mathrm{Cu}$ y los valores de turbiedad. Lo anterior sugiere que, los

Tabla 4. Resultados de las pruebas de correlación de Pearson y de Spearman. Arriba: coeficientes de correlación de Pearson (encima de la diagonal) y coeficientes de correlación de Spearman (debajo de la diagonal). Abajo: valores p inferiores a 0,05 de Pearson (encima de la diagonal) y de Spearman (debajo de la diagonal).

\begin{tabular}{|c|c|c|c|c|c|c|c|c|c|}
\hline & $\mathrm{pH}$ & color & $\mathrm{T}$ & SST & DBO5 & $\mathrm{Cd}$ & $\mathrm{Cu}$ & $\mathrm{Pb}$ & $\mathrm{Zn}$ \\
\hline $\mathrm{pH}$ & 1,00 & 0,97 & & & & & $-0,14$ & $-0,19$ & \\
\hline color & & 1,00 & & & - & & $-0,18$ & - & \\
\hline $\mathrm{t}$ & 0,44 & 0,74 & 1,00 & & & & & & \\
\hline SST & 0,26 & 0,72 & 0,81 & 1,00 & & & & & \\
\hline DBO5 & 0,21 & - & 0,70 & 0,62 & 1,00 & & & & \\
\hline $\mathrm{Cd}$ & $-0,30$ & $-0,15$ & $-0,67$ & $-0,37$ & $-0,53$ & 1,00 & & & \\
\hline $\mathrm{Cu}$ & & & $-0,16$ & $-0,20$ & $-0,70$ & 0,37 & 1,00 & 0,29 & \\
\hline $\mathrm{Pb}$ & & - & $-0,01$ & 0,12 & 0,00 & $-0,20$ & & 1,00 & \\
\hline $\mathrm{Zn}$ & $-0,30$ & 0,18 & 0,40 & 0,57 & 0,58 & $-0,41$ & $-0,23$ & 0,03 & 1,00 \\
\hline & & & & & & & & & \\
\hline & $\mathrm{pH}$ & color & $\mathrm{T}$ & SST & DBO5 & $\mathrm{Cd}$ & $\mathrm{Cu}$ & $\mathrm{Pb}$ & $\mathrm{Zn}$ \\
\hline $\mathrm{pH}$ & - & $<0,01$ & & & & & & & \\
\hline color & & - & & & & & & & \\
\hline $\mathrm{t}$ & 0,01 & 0,03 & - & & & & & & \\
\hline SST & & 0,04 & $<0,01$ & - & & & & & \\
\hline DBO5 & & & 0,02 & & - & & & & \\
\hline $\mathrm{Cd}$ & & & $<0,01$ & & & - & & & \\
\hline $\mathrm{Cu}$ & & & & & 0,02 & & - & & \\
\hline $\mathrm{Pb}$ & & & & & & & & - & \\
\hline $\mathrm{Zn}$ & & & 0,03 & $<0,01$ & & 0,03 & & & - \\
\hline
\end{tabular}


valores de $\mathrm{pH}$, turbiedad y SST, se podrían constituir en indicadores de concentraciones de metales pesados, lo que sería interesante, operacionalmente, por la facilidad de medición de estos parámetros y la prontitud de obtención de resultados, en especial, en lo que concierne al $\mathrm{pH}$ y turbiedad.

Los resultados obtenidos en el marco de este trabajo ponen en evidencia que, a pesar que en la actualidad se utiliza el agua lluvia de escorrentía sobre tejados para satisfacer algunos usos domésticos en zonas periurbanas de Bogotá, no es apta para ninguno de ellos. Lo anterior, se debe, principalmente, a altos valores de turbiedad y altas concentraciones en SST, $\mathrm{DBO}_{5}$ y metales pesados. A excepción del cadmio (Cd), cuyas concentraciones obtenidas son casi diez veces mayores que aquellas reportadas en la literatura, tanto para las concentraciones en SST como en $\mathrm{DBO}_{5}$ y en metales pesados, las concentraciones obtenidas y sus variabilidades son del mismo orden de magnitud que aquellas reportadas en la literatura para aguas lluvias de escorrentía sobre tejados (Gromaire-Mertz et al. 1999; He et al. 2001; Göbel et al. 2007; Schriewer et al. 2008).

Se observaron altas variabilidades, tanto espaciales como temporales, en la calidad de las aguas de escorrentía sobre tejados. Adicionalmente, los resultados variaron en función de los materiales de los techos de las casas en las que se muestreó. Dichas variaciones permiten pensar que en algunos casos y condiciones especiales, el agua lluvia de escorrentía sobre tejados podría ser adaptada para ser utilizada como fuente alternativa para satisfacer algunos usos domésticos, como vaciado de sanitarios y de orinales o lavado de pisos y fachadas. Se piensa, por ejemplo, que aquellas muestras para las cuales se detectó mejor calidad podrían estar asociadas a épocas de lluvias y, por consiguiente, se podría plantear que en algunas épocas del año el agua de escorrentía sobre tejados podría ser utilizada en usos restringidos; sin embargo, se recomienda realizar una caracterización más fina y prolongada en el tiempo de las aguas lluvias de escorrentía sobre tejados, tanto para épocas de tiempo seco como durante épocas de lluvias intensas, de manera a lograr recomendaciones más específicas ligadas a la operación de sistemas de aprovechamiento, en función de las características de las precipitaciones, como el volumen, la intensidad, el caudal y los periodos de tiempo seco anterior.

Los resultados presentados ponen en evidencia la necesidad de tener en cuenta la calidad de las aguas lluvias de escorrentía sobre tejados en zonas periurbanas de las ciudades, para formular políticas y reglamentaciones tendientes a proteger la sanidad civil, en particular, respecto a la agricultura urbana. Se recomienda, sin embargo, un estudio más profundo, con el fin de identificar el origen de la contaminación y proponer soluciones adaptadas a las comunidades que están actualmente utilizando el agua lluvia de escorrentía sobre los tejados, para satisfacer sus necesidades de agua potable. Con este propósito, se prevé realizar campañas experimentales adicionales, entre agosto y diciembre de 2010, las cuales, podrán servir para corroborar relaciones entre contaminantes observadas en el presente trabajo y proponer indicadores de contaminación menos costosos y más oportunos.

Agradecimientos: Los autores agradecen a la Fundación Un Techo para Colombia, a los colaboradores en las comunidades de Soacha y Kennedy y al Laboratorio de Pruebas y Ensayos, de la Facultad de Ingeniería, de la Pontificia Universidad Javeriana. Conflictos de intereses: El manuscrito fue preparado y revisado con la participación de todos los autores, quienes declaramos que no existe ningún conflicto de intereses que ponga en riesgo la validez de los resultados presentados. Financiación: Este estudio fue financiado por la Facultad de Ingeniería de la Pontificia Universidad Javeriana, sede Bogotá.

\section{BIBLIOGRAFÍA}

1. APHA, AWWA, WEF, STANDARD METHODS FOR THE EXAMINATION OF WATER AND WASTEWATER. 1998. $20^{\text {th }}$ ed., American Public Health Association/ American Water Works Association/Water Environment Federation, Washington DC, USA. 1325p.

2. EUROPEAN UNION. 1998. Council Directive 98/83/EC of 3 November 1998 on the quality of water intended for human consumption. Off. J. Eur. Commun. L330, 32-54.

3. EUROPEAN UNION, 2006. Council Directive 2006/7/ EC of of 16 February 2006 on the management of bathing water quality repealing Directive 76/160/EEC. Official Journal of the European Union. L64, 37-51.

4. FLETCHER, T.D.; MITCHELL, V.G.; DELETIC, A. 2007. Is storm water $\mathrm{H}+$ harvesting beneficial to urban waterway environmental flows? Wat. Sci. Tech. (UK). 55(4):265-272.

5. GHISI, E.; TAVARES, D.; ROCHA, V. 2009. Rainwater harvesting in petrol stations in Brasilia: Potential for potable water savings and investment feasibility analysis. Resources, Conservation and Recycling (NTH). 54(2):79-85. 
6. GÖBEL, P.; DIERKES, C.; COLDEWEY, W.G. 2007. Storm water runoff concentration matrix for urban areas. J. Contaminant Hydrology (USA). 91(1-2):2642.

7. GROMAIRE-MERTZ, M.C.; GARNAUD, S.; GONZALEZ, A.; CHEBBO, G. 1999. Characterization of urban runoff pollution in Paris. Wat. Sci. Tech. (UK). 39(2):18.

8. HAN, M.; Ki, J. 2010. Establishment of sustainable water supply system in small islands through rainwater harvesting (RWH): case study of Guja-do. Wat. Sci. Tech. (UK). 62(1):148-153.

9. HATT, B.E.; DELETIC, A.; FLETCHER, T.D. 2006. A review of integrated storm water treatment re-use in Australia. J. Environ. Manage. 76:102-113.

10. HE, W.; ODNEVALL-WALLINDER, I.; LEYGRAF, C. 2001. A comparison between corrosion rates and runoff rates from new and aged copper and zinc as roofing material. Water, Air, and Soil Pollution: Focus (NTH). 1:67-82.

11. LAZAROVA, V.; PAPADOPOULOS, I.; BAHRI, A. 2004. Code of Successful Agronomic Practice. En: Lazarova, V.; Bahri, A. eds. Water Reuse for Irrigation: Agriculture, Landscapes, and Turf Grass. Ed. CRC Press (Boca Raton). p.103-150.

12. MCCARTHY, D.; DELETIC, A.; MITCHELL, V.; FLETCHER, T.; DIAPER, C. 2008. Uncertainties in stormwater $E$. coli levels. Water Res. (Holanda). 42(67):1812-1824. 13. MINISTERIO DE LA PROTECCIÓN SOCIAL,
MINISTERIO DE AMBIENTE VIVIENDA Y DESARROLLO TERRITORIAL. 2007. Por medio de la cual se señalan características, instrumentos básicos y frecuencias del sistema de control y vigilancia para la calidad del agua para consumo humano. Resolución 2115 de 2007, Bogotá, D.C.: Ministerio de la Protección Social; Ministerio de Ambiente Vivienda y Desarrollo Territorial.
14. MINISTERIO DE SALUD REPÚBLICA DE COLOMBIA. 1984. Decreto 1594. Usos del agua y residuos líquidos. 52p.

15. MITCHELL, V.G.; MCCARTHY, D.T.; DELETIC, A.; FLETCHER, T.D. 2008. Urban stormwater harvesting - sensitivity of a storage behaviour model. Environ. Modelling \& Software (Holanda). 23(6):782-793.

16. PACHÓN QUINCHE, J.E. 2005. Desarrollo de una metodología para la estimación de emisiones de partículas en cuatro municipios de Cundinamarca. Material Particulado Atmosférico y Salud. ed: Uniandes (Colombia). 1:41-54.

17. R DEVELOPMENT CORE TEAM. 2010. R: A language environment for statistical computing. $\mathrm{R}$ Foundation for Statistical Computing, Vienna, Austria. ISBN 3-900051-07-0, URL disponible desde Internet en http://www.R-project.org. (con acceso el 10/06/2010).

18. SCHRIEWER, A.; HORN, H.; HELMREICH, B. 2008. Time focused measurements of roof runoff quality. Corros Sci. (UK). 50:384-391.

19. SCHUELER, T.R.; HOLLAND, H.K. 2000. Is Rooftop Runoff Really Clean? The Practice of Watershed Protection. Center for Watershed Protection, Ellicott City, MD (USA). 12:84-85.

20. U.S. EPA. 2004. Guidelines for Water Reuse, EPA625/R-04-108, U.S. Environmental Protection Agency U.S. Agency for International Development, Washington, DC. 450p.

21. WHO. 2006. Guidelines for the safe use of wastewater, excreta and greywater. Volume 2. Wastewater use in agriculture. World Health Organization, Geneva (Suiza). - xxii, 196p.

Recibido: Septiembre 21 de 2010

Aceptado: Marzo 2 de 2011 